PROCEEDINGS OF THE

AMERICAN MATHEMATICAL SOCIETY

Volume 139, Number 4, April 2011, Pages 1409-1410

S 0002-9939(2010)10668-8

Article electronically published on August 30, 2010

\title{
A NON-RESIDUALLY SOLVABLE HYPERLINEAR ONE-RELATOR GROUP
}

\author{
JON P. BANNON \\ (Communicated by Marius Junge)
}

\begin{abstract}
In this short paper, we prove that the group $\left\langle a, b \mid a=\left[a, a^{b}\right]\right\rangle$ is hyperlinear. Unlike the nonresidually finite Baumslag-Solitar groups, this group is not residually solvable.
\end{abstract}

\section{INTRODUCTION}

Let $\Gamma$ denote the one-relator group $\left\langle a, b \mid a^{-1}\left[a, a^{b}\right]\right\rangle$, where $a^{b}=b a b^{-1}$ and $\left[a, a^{b}\right]=a^{-1}\left(a^{b}\right)^{-1} a a^{b}$. This group was introduced by G. Baumslag in Baum69] as an example of a noncyclic one-relator group with the property that all of its finite index quotients are cyclic. It follows that the group $\Gamma$ is not residually finite. Also, $\Gamma$ is not residually solvable, since $a$ lies in every one of the derived subgroups of $\Gamma$. A countable discrete group $G$ is hyperlinear if it can be embedded as a subgroup of the unitary group $U\left(\mathcal{R}^{\omega}\right)$ of an ultrapower $\mathcal{R}^{\omega}$ of the hyperfinite type $I I_{1}$ factor $\mathcal{R}$ (cf. Pest08]). Equivalently, $G$ is hyperlinear if the group von Neumann algebra $L(G)$ is embeddable into $\mathcal{R}^{\omega}$ (cf. Pest08). Proposition 4.14 of [Ueda09] establishes that every HNN extension of an $\mathcal{R}^{\omega}$-embeddable type $I I_{1}$ factor over a hyperfinite von Neumann subalgebra is also $\mathcal{R}^{\omega}$-embeddable. We use this fact along with a now standard trick of $\mathrm{McCool}$ and Schupp for one-relator groups to prove that the group $\Gamma$ above is hyperlinear. The main interest in this example is that it is an example of a nonresidually solvable hyperlinear one-relator group, and thus our result sheds a little light on the question of Nate Brown asking whether every onerelator group is hyperlinear. In $\operatorname{Rad} 00$, Radulescu proved that the nonresidually finite Baumslag-Solitar group $\left\langle a, b \mid a b^{3} a^{-1} b^{-2}\right\rangle$ is hyperlinear. Radulescu's result is shown in Pest08] to follow more simply from the fact that these Baumslag-Solitar groups are residually solvable, and hence sofic.

\section{MAin RESUlt}

Theorem 2.1. The group $\Gamma=\left\langle a, b \mid a^{-1}\left[a, a^{b}\right]\right\rangle$ is hyperlinear.

Proof. We apply a rewriting process due to McCool and Schupp (cf. McCSch73). Let $a_{0}=a$ and $a_{-1}=b a b^{-1}$. Note that the word

$$
a^{-1}\left[a, a^{b}\right]=a^{-2} b a^{-1} b^{-1} a b a b^{-1}
$$

Received by the editors February 17, 2010 and, in revised form, April 26, 2010.

2010 Mathematics Subject Classification. Primary 46L10; Secondary 20F65.

Key words and phrases. Sofic group, hyperlinear group, one-relator group.

(C)2010 American Mathematical Society 
when rewritten in terms of $a_{0}$ and $a_{-1}$ becomes

$$
a_{0}^{-2}\left(a_{-1}\right)^{-1} a_{0}\left(a_{-1}\right) .
$$

The group $H=\left\langle a_{0}, a_{-1} \mid a_{0}^{-2}\left(a_{-1}\right)^{-1} a_{0}\left(a_{-1}\right)\right\rangle$ is amenable, essentially by the Tits alternative. Or, we may appeal to Theorem 1.2 of CeGrig97 and note that $a_{0}^{-2}\left(a_{-1}\right)^{-1} a_{0}\left(a_{-1}\right)$ has exponent sum zero on $a_{-1}$ and can be obtained from $\left(a_{-1}\right) a_{0}\left(a_{-1}\right)^{-1} a_{0}^{-2}$ by inverting $a_{-1}$ and cyclically shifting, and hence $H$ is amenable. We then note that the group $\Gamma$ is isomorphic to the HNN extension

$$
H *_{\varphi}=\left\langle t, H \mid t^{-1} a_{-1} t=a_{0}\right\rangle .
$$

Now, consider the group von Neumann algebra $L\left(H *_{\varphi}\right)$. By Corollary 3.5 of Ueda05, this is isomorphic to a reduced HNN extension of the hyperfinite $I I_{1}$ factor $\mathcal{R}$ over $L(\mathbb{Z})$. Therefore, by Proposition 4.14 of [Ueda09, $L\left(H *_{\varphi}\right)$ is embeddable into an $\mathcal{R}^{\omega}$, and therefore $\Gamma$ is hyperlinear.

Remark 2.2. We wish to thank the referee for pointing out that recently it has been shown that any HNN extension of a sofic group over an amenable subgroup is sofic. Precisely, this is Corollary 3.4 of DykCol10. We may, in the above proof, replace Ueda's result by this one and obtain that $\Gamma$ is, in fact, a sofic group.

\section{REFERENCES}

[Baum69] G. Baumslag, A non-cyclic one-relator group all of whose finite quotients are cyclic, J. Austral. Math. Soc. 10 (1969), 497-498. MR0254127 (40:7337)

[CeGrig97] T.G. Ceccherini-Silberstein and R. I. Grigorchuk, Amenability and growth in onerelator groups, L'Enseignement Mathématique (2) 43 (1997), 337-354. MR1489891 (99b:20057)

[DykCol10] K. Dykema and B. Collins, Free products of sofic groups with amalgamation over amenable groups, arXiv:math/1003.1675v1, 2010.

[McCSch73] J. McCool and P. Schupp, On one relator groups and HNN extensions, J. of the Austral. Math. Soc. 16 (1973), 249-256. MR0338186 (49:2952)

[Pest08] V. Pestov, Hyperlinear and sofic groups: a brief guide, Bull. Symbolic Logic 14, no. 4 (2008), 449-480. MR2460675 (2009k:20103)

[Rad00] F. Rădulescu, The von Neumann algebra of the non-residually finite Baumslag group $\left\langle a, b \mid a b^{3} a^{-1}=b^{2}\right\rangle$ embeds into $R^{\omega}$. Theta Ser. Adv. Math., vol. 9, Theta, Bucharest, 2008. MR2436761 (2009k:46110)

[Ueda05] Y. Ueda, HNN extensions of von Neumann algebras, Journal of Functional Analysis 225 , no. 2 (2005), 383-426. MR2152505 (2006k:46100)

[Ueda09] Y. Ueda, Remarks on HNN extensions in operator algebras, Illinois J. Math. 52, no. 3 (2008), 705-725. MR2546003 (2010h:46093)

Department of Mathematics, Siena College, Loudonville, New York 12211

E-mail address: jbannon@siena.edu 